\title{
Structural Role of Hydrophobic Core in Proteins-Selected Examples
}

\author{
Banach $\mathbf{M}^{1,2}$, Kalinowska $B^{1,2}$, Konieczny $L^{3}$ and Roterman $I^{1 *}$ \\ ${ }^{1}$ Department of Bioinformatics and Telemedicine, Jagiellonian University Medical College, Poland \\ ${ }^{2}$ Faculty of Physics, Astronomy and Applied Computer Science, Jagiellonian University, Poland \\ ${ }^{3}$ Chair of Medical Biochemistry, Jagiellonian University Medical College, Poland
}

\begin{abstract}
This paper discusses the sequence/structure relation. The core question concerns the degree to which similar sequences produce similar structures and vice versa. A mechanism by which similar sequences may result in dissimilar structures is proposed, based on the Fuzzy Oil Drop (FOD) model in which structural similarity is estimated by analyzing the protein's hydrophobic core. We show that local changes in amino acid sequences, in addition to producing local structural alterations at the substitution site, may also change the shape of the hydrophobic core, significantly affecting the overall tertiary conformation of the protein. Our analysis focuses on four sets of proteins: 1) Pair of designer proteins with specially prepared sequences; 2) Pair of natural proteins modified (mutated) to converge to a point of high-level sequence identity while retaining their respective wild-type tertiary folds; 3 ) Pair of natural proteins with common ancestry but with differing structures and biological profiles shaped by divergent evolution; and 4) Pair of natural proteins of high structural similarity with no sequence similarity and different biological function.
\end{abstract}

Keywords: Structure comparison; Structure alignment; Structural differences; Structure prediction; Evolution; Protein folding; Hydrophobicity

\section{Introduction}

The presented analysis concerns the well-known problem of correlating the protein's amino acid sequence with its 3D structure [14]. The search for algorithms which can be used to translate the former into the latter is a fundamental problem in proteomics $[5,6]$ and often yields useful insight into the specific properties of individual proteins [7].

A classic example of this phenomenon is the group of structures referred to as immunoglobulin-like domains. Such domains are present in all immunoglobulins (where they determine their function) but are also encountered in enzymes and transport proteins [810]. Immunoglobulins exhibit high structural similarity, adopting characteristic "sandwich" conformations with rather low sequence similarity. Even among immunoglobulins domains the $\lambda$ and $\kappa$ sequences are identified. Of course, the diversity of proteins which are not immunoglobulins but which do contain immunoglobulin-like domains is even greater [10].

In addition to the above, studies have revealed cases where very similar sequences produce significantly different structural forms [11]. For example, the KGVVPQLVK sequence generates a classic $\beta$-twist in 1 PKY but adopts a helical conformation in 1IAL [12-14]. The three 7-residue sequences which also share this property of different secondary structure for identical sequences are given in Jacoboni et al. [15].

Conservative hydropathic identity at geometrically equivalent positions is the object of analysis in Krissinel [16]. Our work focuses on structural differences in four pairs of proteins: 1) Pair of designer proteins with specially prepared sequences; 2) Pair of natural proteins modified (mutated) to converge to a point of high-level sequence identity while retaining their respective wild-type tertiary folds; 3 ) Pair of natural proteins with common ancestry but with differing structures and biological profiles shaped by divergent evolution; and 4) Pair of natural proteins of high structural similarity with no sequence similarity and different biological function.

In attempting to show the role of hydrophobic core structure in structural stabilization we refer to the Fuzzy Oil Drop (FOD) model, which predicts the $3 \mathrm{D}$ conformation of the target protein by simulating the emergence of a hydrophobic core. While our research has identified some interesting correlations, generalizing them remains an open issue: in order to determine whether the presented results may, in fact, be generalized we need to process a much larger database of proteins.

\section{Materials and Methods}

\section{Data}

The presented analysis concerns four sets of two proteins each. The first set comprises two de novo designed proteins with a sequential similarity of $88 \%$ but with differing $3 \mathrm{D}$ structures. The second describes two natural proteins which are modified (mutated) in a stepwise fashion in order to align their sequences while preserving structural differentiation (helix-to-Beta). The third set includes two natural homologues with common ancestry. The fourth one discusses pair of natural proteins of high structural similarity with no sequence similarity and different biological function.

\section{De novo design}

Two de novo designed proteins, 2JWU and 2JWS, also referred to as $G_{A} 88$ and $G_{B} 88$ share $88 \%$ sequence identity but different folds [17]. The geometries of the seven nonidentical residues (of 56 total) provide insight into the structural basis for switching between 3- $\alpha$ and $\alpha / \beta$ conformations. Further mutation of a subset of these no identities, guided by the $G_{A} 88$ and $G_{B} 88$ structures, leads to proteins

*Corresponding author: Roterman I, Department of Bioinformatics and Telemedicine, Łazarza 16, 31-530 Krakow, Poland, Tel: 48126199693; E-mail: myroterm@cyf-kr.edu.pl

Received October 14, 2016; Accepted November 06, 2016; Published November 11, 2016

Citation: Banach M, Kalinowska B, Konieczny L, Roterman I (2016) Structural Role of Hydrophobic Core in Proteins-Selected Examples. J Proteomics Bioinform 9: 276-286. doi: 10.4172/jpb. 1000416

Copyright: ( 2016 Banach M, et al. This is an open-access article distributed under the terms of the Creative Commons Attribution License, which permits unrestricted use, distribution, and reproduction in any medium, provided the original author and source are credited. 
with even higher levels of sequence identity (95\%) and differing folds. Thus, conformational switching to an alternative monomeric fold of comparable stability can be effected with just a handful of mutations in a small protein. This result has implications for understanding not only the folding code but also the evolution of new folds. The CATH [18] classification for these two proteins is as follows: 1.10.8.40- Mainly Alpha Orthogonal Bundle for 2JWS and 3.10.20.10.-R-Alpha Beta Roll for $2 \mathrm{JWU}$.

\section{Wild type proteins with aim-oriented mutations}

Two proteins: G311 (1ZXG) and A219 (1ZXH) are modified versions of wild-type proteins designated $G$ and A (IgG binding domains, source: Staphylococcus aureus for 1ZXG and Streptococcus $s p$. for 1ZXH) respectively [19]. The series of mutations aimed at achieving a high level of sequence identity while preserving wild-type 3D structures. Both proteins (G311 vs. G and A219 vs. A) represent backbone RMS-D 1.4 $\AA$, maintaining wild-type secondary structures: $\alpha / \beta$ for G311 and helical for A219. The final sequence identity of both modified proteins is on the level of $59 \%$. All relevant data was taken from a paper describing experimental results of protein modifications [19].

\section{Homologous proteins}

The next pair comprises two proteins with common ancestry but different folds and functions: 2PIJ (Pfl 6 BETA) (source: Pseudomonas fluorescens) Cro protein from prophage pfl 6 in P. fluorescens pf-5 [20] and 3BD1 (Xfaso 1) Cro protein from putative prophage element $X$. fastidiosa strain ann-1 [20] (source: Xylella fastidiosa). Both proteins share an identical CATH classification: 1.10.260.40-Mainly Alpha Orthogonal Bundle.

The differences between homologous proteins are due to evolutionary pressure. In the presented case the sequence identity is $40 \%$, yet the $\alpha$-helix is replaced by a $\beta$-sheet in the C-terminal region spanning approximately 25 residues. According to Roessler et al. [20], sedimentation analysis suggests a correlation between helix-to-sheet conversions, along with strengthened dimerization.

Unrelated proteins of similar fold: Two proteins not related in respect neither to evolution nor to function representing however the common fold classified by CATH as $3.40 .50 .360-3 \mathrm{CHY}$ (signal transduction protein) [21] and 3.40.50.2300-1RCF (flavodoxin) [22] both of 3-layer (aba) sandwich form with no sequence similarity (about 19\%).

\section{Introduction to the Fuzzy Oil Drop (FOD) model}

The Fuzzy Oil Drop (FOD) model is a modification of the previously described oil drop model which asserts that hydrophobic residues tend to migrate to the center of the protein body while hydrophilic residues are exposed on its surface $[23,24]$. The FOD replaces the binary discrete model [25] with a continuous function peaking at the center of the molecule [23], which causes hydrophobicity density values to decrease along with distance from the center, reaching zero on the molecular surface. The idealized, theoretical hydrophobicity distribution is expressed by 3D Gauss function. The size of molecule shall be expressed by sigma parameters for Gauss function. The characteristics of this function allows represent the hydrophobicity distribution with maximum in the center of ellipsoid with decrease together with the increase of distance versus the center reaching zero level in the distance equal to 3 sigma in any direction. This idealized distribution ensures high solubility since the entire ellipsoid is covered by the hydrophilic shell.

On the other hand the actual distribution of hydrophobicity density observed in a protein molecule depends on inter-chain interactions, which, in turn, depend on the intrinsic hydrophobicity of each amino acid. Intrinsic hydrophobicity can be determined by experimental studies or theoretical reasoning- our work bases on the scale published in Kalinowska et al. [24] while the force of hydrophobic interactions has been calculated using other scales as it was shown in Kalinowska et al. [24]. For each amino acid $j$ (or, more accurately, for each effective atom) the sum of interactions with its neighbors is computed and subsequently normalized by dividing it by the number of elementary interactions (following the function proposed in Levitt [26].

The two hydrophobicity density distribution profiles: the expected (T) and observed $(\mathrm{O})$ distribution an be compared quantitatively. Quantitative expressing of the differences between the expected $(\mathrm{T})$ and observed $(\mathrm{O})$ distribution is possible using the Kullback-Leibler divergence entropy formula [27]:

$$
D_{K L}\left(p \mid p^{0}\right)=\sum_{i=1}^{N} p_{i} \log _{2}\left(p_{i} / p_{i}^{0}\right)
$$

The value of $\mathrm{D}_{\mathrm{KL}}$ expresses the distance between the observed ( $\mathrm{p}$ ) and target $\left(\mathrm{p}_{0}\right)$ distributions, the latter of which is given by the $3 \mathrm{D}$ Gaussian (T). The observed distribution ( $\mathrm{p}$ ) is referred to as $\mathrm{O}$.

For the sake of simplicity, we introduce the following notation:

$$
O \mid T=\sum_{i=1}^{N} O_{i} \log _{2}\left(O_{i} / T_{i}\right)
$$

Since $\mathrm{D}_{\mathrm{KL}}$ is a measure of entropy it must be compared to a reference value. In order to facilitate meaningful comparisons, we have introduced another opposite boundary distribution (referred to as "uniform" or R) which corresponds to a situation where each effective atom possesses the same hydrophobicity density $(1 / N$, where $N$ is the number of residues in the chain). This distribution is deprived of any form of hydrophobicity concentration at any point in the protein body:

$$
O \mid R=\sum_{i=1}^{N} O_{i} \log _{2}\left(O_{i} / R_{i}\right)
$$

Comparing $\mathrm{O} \mid \mathrm{T}$ and $\mathrm{O} \mid \mathrm{R}$ tells us whether the given protein $(\mathrm{O})$ more closely approximates the theoretical (T) or uniform (R) distribution. Proteins for which $\mathrm{O}|\mathrm{T}>\mathrm{O}| \mathrm{R}$ are regarded as lacking a prominent hydrophobic core. To further simplify matters we introduced the following Relative Distance (RD) criterion:

$$
R D=O \mid T /(O|T+O| R)
$$

$\mathrm{RD}<0.5$ is understood to indicate the presence of a hydrophobic core. Figure 1 presents a graphical representation of RD values, restricted (for simplicity) to a single dimension.

$\mathrm{D}_{\mathrm{KL}}$ (as well as $\mathrm{O}|\mathrm{T}, \mathrm{O}| \mathrm{R}$ and $\mathrm{RD}$ ) may be calculated for specific structural units (protein complex, single molecule, single chain, selected domain etc.) In such cases the bounding ellipsoid is restricted to the selected fragment of the protein. It is also possible to determine the status of polypeptide chain fragments within the context of a given ellipsoid. This procedure requires prior normalization of $\mathrm{O} \mid \mathrm{T}$ and $\mathrm{O} \mid \mathrm{R}$ values describing the analyzed fragment.

$\mathrm{RD}$ can be calculated for entire units (protein, chain, domain) and for selected fragment (following normalization of $T_{i}$ and $\mathrm{O}_{i}$ values of the fragment under consideration).

The above procedure will be applied in the analysis of proteins described in this paper. By restricting our analysis to individual fragments, we can determine whether a given fragment participates in the formation of a hydrophobic core. In particular, fragments of chain representing well defined secondary folds which satisfy $\mathrm{RD}<0.5$ are 
thought to contribute to structural stabilization, while fragments for which $\mathrm{RD} \geq 0.5$ are less stable. Such fragments, if present on the surface of the protein, may potentially form complexation sites. The fragments of chains are defined by their secondary structure. Identification of secondary structural folds and the composition of protein domains follow the CATH [18] and PDBsum [28] classifications. Likewise, interdomain/inter-chain contacts have been identified on the basis of the PDBsum distance criteria [28].

The graphic presentation of RD interpretation is shown in Figure 1.

The OORF system of RD calculation uses the method from ORF calculation in DNA analysis. OORF stands from Overlapped Open Reading Frame. The window of declared size (10 aa in our analysis) is taken as the fragment, the RD value is calculated. For example fragment $1-10$ gets described by its RD value. Then the next window (2-11 aa) is taken for $\mathrm{RD}$ calculation. The $\mathrm{RD}$ value for each window requires prior normalization (the sum of $\mathrm{T}_{i}$ and $\mathrm{O}_{i}$ belonging to the window shall be equal to 1.0). This form of calculation makes possible characteristics of entire chain regardless the secondary structure.

The detailed description of the FOD model is available in the paper recently published [29].

\section{Results}

\section{De novo designed proteins}

According to results given in Table 1 the structure of $2 \mathrm{JWS}\left(\mathrm{G}_{\mathrm{A}} 88\right)$ is consistent with the model both as a whole and in its packed section (without the N-terminal fragment 1-7 which was eliminated from calculation since the FOD model works well with globular proteins). This operation does not affect the status of helical folds.

In $2 J W U\left(G_{B} 88\right)$ four $\beta$-folds can be distinguished, in addition to a single helix. This molecule also contains a loop (38-41). The $\beta$-fragment at $42-46$ and the loop both diverge from the model even though in $G_{A} 88$ the same residues form parts of an accordant helix. The fragment at $38-46$ is characterized by higher-than-expected hydrophobicity density (Figure 2A). Since this fragment is exposed on the protein surface (expected hydrophobicity is low), it may be responsible for possible forming complexes with other proteins which also expose hydrophobic areas on their surface.

The consistently high accordance of 2JWS-both as a whole and when subdivided into folds-suggest a relative lack of local deformations. It may be speculated that as predicted by the FOD model, this molecule is highly water-soluble with low tendency to interact with any ligand molecule.

Figures $2 \mathrm{~A}$ and $2 \mathrm{D}$ present the hydrophobicity density profiles for both proteins, showing the values ascribed to each residue in the polypeptide chain. The distinguished fragments satisfy the condition of high expected and high observed hydrophobicity in both molecules. From the point of view of the model both molecules contain well-defined hydrophobic cores. One shall notice that the FOD model identifies the central part of molecule as the hydrophobic core together with the shell of intermediate coat including the exposed surface of expected hydrophobicity close to zero (hydrophilic surface). The co-existence of these two parts makes the hydrophobic core complete as protected and isolated against immediate contact with water environment. The identification of residues recognized as hydrophobic core members is based on the high expected and high observed hydrophobicity. Residues following this condition are recognized as responsible for hydrophobic core construction.

The profiles shown in Figures $2 \mathrm{~B}$ and $2 \mathrm{C}$ (OORF distributions) reveal significant differences pointing different fragments of low $\mathrm{RD}$ values suggesting high accordance between expected and observed distributions in both proteins.

The observations listed above seem to support the conclusion that both proteins are structurally different in terms of their hydrophobic

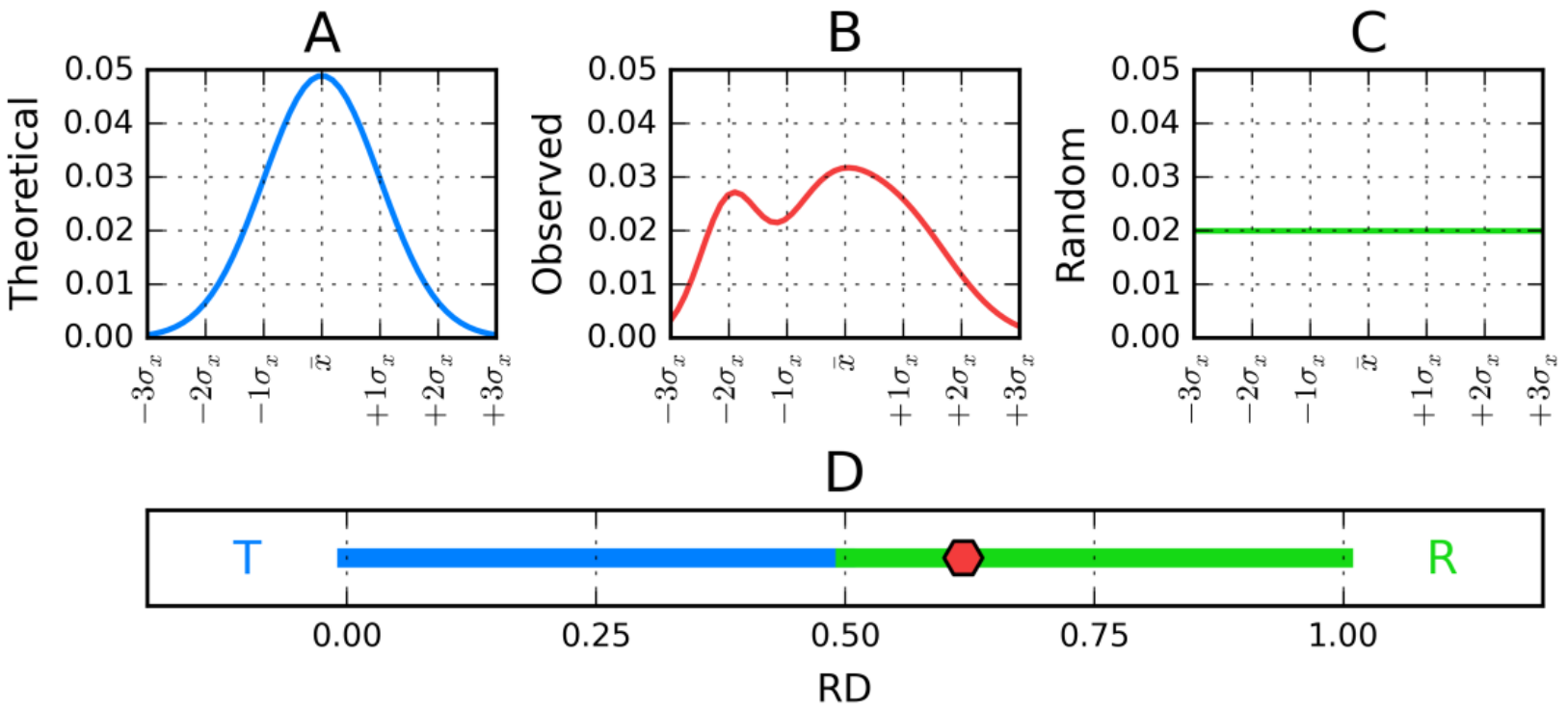

Figure 1: Graphical representation of fuzzy oil drop model hydrophobicity distributions obtained for a hypothetical protein reduced to a single dimension for simplicity. A) Theorized Gaussian distribution (blue) while the chart $\mathrm{C}$ corresponds to the uniform distribution (green). Actually observed (red) hydrophobicity density distribution in the target protein $\mathrm{B}$, while its corresponding value of RD (relative distance), and in $\mathrm{D}$ is marked on the horizontal axis with a red diamond. According to the fuzzy oil drop model this protein does not contain a well-defined hydrophobic core, because its RD value, equal to 0.619 , is above the 0.5 threshold (or-generally-closer to $\mathrm{R}$ than $\mathrm{T}$ ). 
cores. The construction of hydrophobic core in 2JWS is generated by central part of polypeptide chain, comprising two fragments (Figure 2A) while in $2 J W U$, it requires three separate fragments to participate in core generation (Figure 2D).

At this point it might be interesting to speculate about the progress of the folding process in each of these two cases. In 2JWS the hydrophobic core nucleates near the center of the chain, with the remaining sections aligning themselves to the emerging core. While in 2JWU the nucleation is mainly constructed by $\mathrm{N}$ - and C-terminal fragments with the participation also of central fragment of the chain.

Figure 3 presents a comparison of the status of each fold in both proteins, indicating mutations and the correspondence between the observed and theoretical hydrophobicity density in each fold. The makeup of the hydrophobic core is also depicted.

In summary, the introduction of seven mutations (G24A, I25T, I30F, I33Y, L45Y, I49T, L50K-with 2JWS serving as the reference strain) results in a far higher concentration of hydrophobic residues in 2JWS. This enlarges the hydrophobic core which is formed by the central fragment of the polypeptide chain. Unlike 2JWS, in 2JWU the core is made up of three separate fragments, including one that forms part of the shell (with lower hydrophobicity density). Substitutions at G42A, I25T, I30F, I33Y, L45Y result in the appearance of a long fragment which forms part of the hydrophobic core, while the presence of $\mathrm{Y}, \mathrm{T}$ and $\mathrm{K}$ in the C-terminal fragment of 2JWU causes a hydrophobicity density gradient to emerge in the surface zone where hydrophilic residues appear, in accordance with the theoretical model. Figure 3 shows clearly the influence of mutations since the residues changed concern the positions of the central part in 2JWU. The location of these residues in 2JWU is rather distributed. In consequence different fragments of the chain participate in hydrophobic core formation and one fragment (the $\beta$-structural fragment) appears to represent the hydrophobicity density distribution discordant versus the idealized one.

\section{Wild type proteins with aim-oriented mutations}

The results listed in Table 2 indicate very high agreement with the FOD model in two compared proteins: $1 \mathrm{ZXH}$ and $1 \mathrm{ZXG}-$ two IgG binding domains. Additionally, each secondary structure (including loops) remains consistent with structural predictions provided by the model.

Considering the large set of molecules analyzed using the FOD model we can conclude that the presented proteins are among the most accordant in the entire set, as indicated by their $\mathrm{RD}$ values (so far $\mathrm{RD}=0.38$ for the immunoglobulin-like domain in titin (1TIT) was found to be the lowest). The structure of the hydrophobic core, which is understood as the entire tertiary conformation of the protein (including the core itself and its hydrophilic sheath) remains highly consistent with theoretical predictions, as shown in Figures $4 \mathrm{~A}$ and $4 \mathrm{~B}$. Figures $4 \mathrm{C}$ and $4 \mathrm{D}$ illustrate the agreement between theoretical and observed hydrophobicity density distributions, with correlation coefficients of 0.847 and 0.784 for G311 and A219 respectively. The figures also reveal highly hydrophobic (core) and hydrophilic (surface) residues, whose placement can be seen in Figure 5A. Finally, Figure 5B marks the loci of point mutations-though the affected residues do not clearly belong either to the core or to the hydrophilic sheath. Figure 5 visualizes the positions of mutations and their influence on the hydrophobic core rearrangement.

Analysis of results for G311 and A219 indicates that tertiary structural stabilization (by hydrophobic core) appears to be dependent on a proper distribution of hydrophobicity density, ensuring the presence of a highly hydrophobic core as well as the encapsulating hydrophilic sheath, with near-zero hydrophobicity density values on its surface. Unfortunately the authors of the cited experimental work [17] do not report on the relation between the introduced mutations and the proteins' capability to bind immunoglobulins. From the point of view of the FOD model, however, the mutated molecules should be less prone to complexation than their wild-type counterparts. This supposition follows from the observed good agreement between the theoretical and observed hydrophobicity density distributions-note that complexation sites are typically characterized by marked differences between both profiles (theoretical and observed). According to the model, a protein which only exposes hydrophilic residues on its surface should be highly soluble and incapable of interacting with any ligands other than dissolved ions. This phenomenon is evidenced by antifreeze and down-

\begin{tabular}{|c|c|c|c|c|}
\hline & SECONDARY FRAGMENT & 이T & O|R & RD \\
\hline \multirow[t]{3}{*}{$2 J W U\left(G_{B} 88\right)$} & COMPLETE MOLECULE & 0.126 & 0.249 & 0.335 \\
\hline & B 1-9 & 0.052 & 0.157 & 0.249 \\
\hline & B 12-20 & 0.196 & 0.248 & 0.441 \\
\hline \multirow[t]{2}{*}{ Mut-4 } & H 22-37 & 0.146 & 0.203 & 0.419 \\
\hline & L 38-41 & 0.078 & 0.069 & 0.533 \\
\hline Mut-1 & B $42-46$ & 0.203 & 0.160 & 0.558 \\
\hline Mut-1 & B $50-56$ & 0.066 & 0.180 & 0.268 \\
\hline \multirow[t]{2}{*}{ 2JWS $\left(G_{A} 88\right)$} & COMPLETE MOLECULE & 0.182 & 0.308 & 0.371 \\
\hline & Unstructure 1-7 & 0.235 & 0.241 & 0.493 \\
\hline Mut-1 & H 8-24 & 0.260 & 0.322 & 0.447 \\
\hline Mut-2 & H 26-35 & 0.055 & 0.251 & 0.181 \\
\hline Mut-3 & H 38-52 & 0.129 & 0.179 & 0.418 \\
\hline $\begin{array}{c}\text { 2JWS }\left(G_{A} 88\right) \\
8-56\end{array}$ & $\begin{array}{l}\text { COMPLETE } \\
\text { DOMAIN }\end{array}$ & 0.170 & 0.277 & 0.380 \\
\hline Mut-1 & H 8-24 & 0.213 & 0.328 & 0.394 \\
\hline Mut-2 & H 26-35 & 0.078 & 0.250 & 0.238 \\
\hline Mut-3 & H 38-52 & 0.161 & 0.175 & 0.479 \\
\hline
\end{tabular}

Table 1: Properties of selected proteins from the point of view of the fuzzy oil drop model. O|T values indicate the distance between the observed and theoretical distribution while O|R values show the corresponding distance between the observed and uniform distribution. RD values are in relation to the idealized (theoretical) distribution. Each parameter is listed for complete molecules and for their individual folds. The leftmost column also includes information on sequential discrepancies between pairs of molecules. Mut-N indicates an exchange and the number of affected residues (2JWS vs. 2JWU) in each fragment. The bottom part of the table shows results for the packed domain of 2JWS (without the unstructured $\mathrm{N}$-terminal fragment). B stands for $\beta$-type structures while $\mathrm{H}$ indicates a right-handed $\alpha$-helix. 

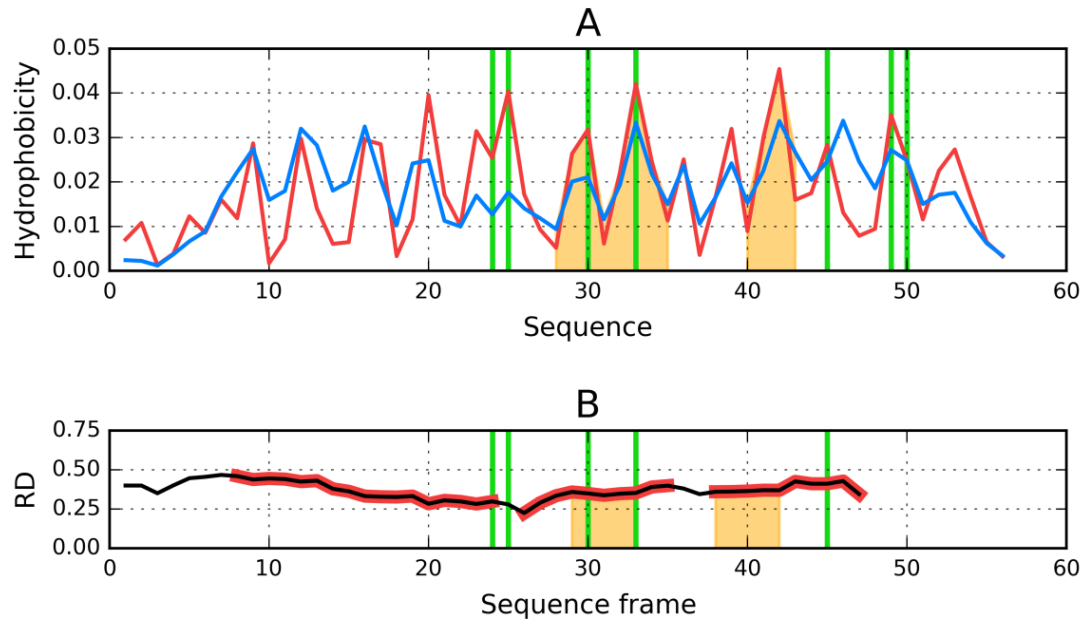

C

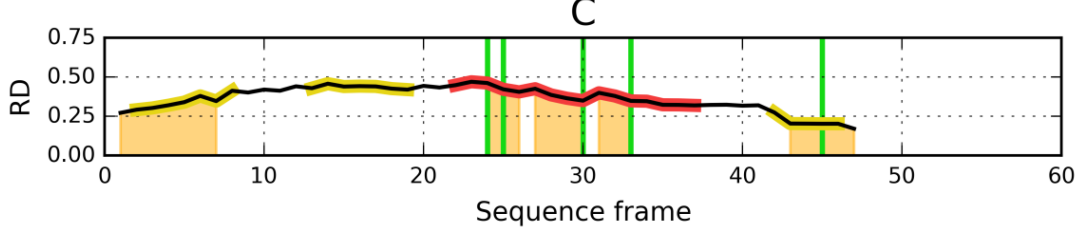

D

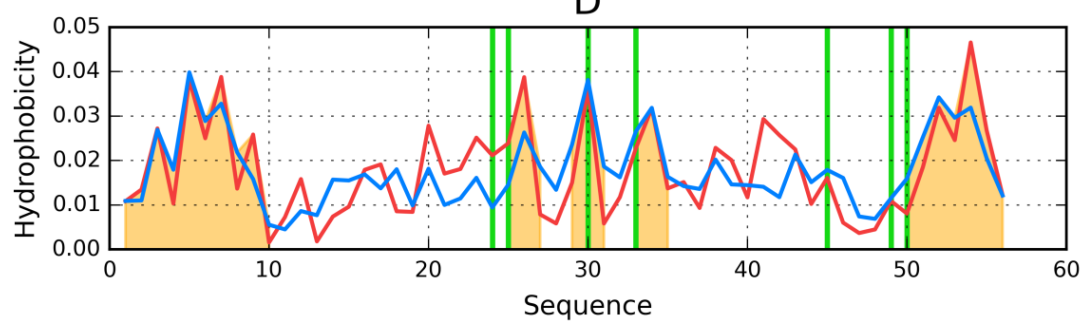

Figure 2: Hydrophobicity density distribution profiles for two molecules (de novo design) differing with respect to their secondary structure: A, B-2JWS (G 88), C, D-2JWU $\left(G_{8} 88\right)$. Theoretical hydrophobicity profile is plotted in blue while that of observed hydrophobicity is red. Vertical green lines localize positions of mutations. Orange shades under the profiles indicate positions of hydrophobic core, understood as groups of residues with peak values of both hydrophobicity distributions. Figures $2 \mathrm{~B}$ and $2 \mathrm{C}$ present RD values of both proteins calculated in the OORF (Overlapped Open Reading Frame) system with 10 aa window size. Red and yellow fragments visible on those figures denote locations of secondary structure fragments: $\alpha$-helices and $\beta$-sheets, respectively.

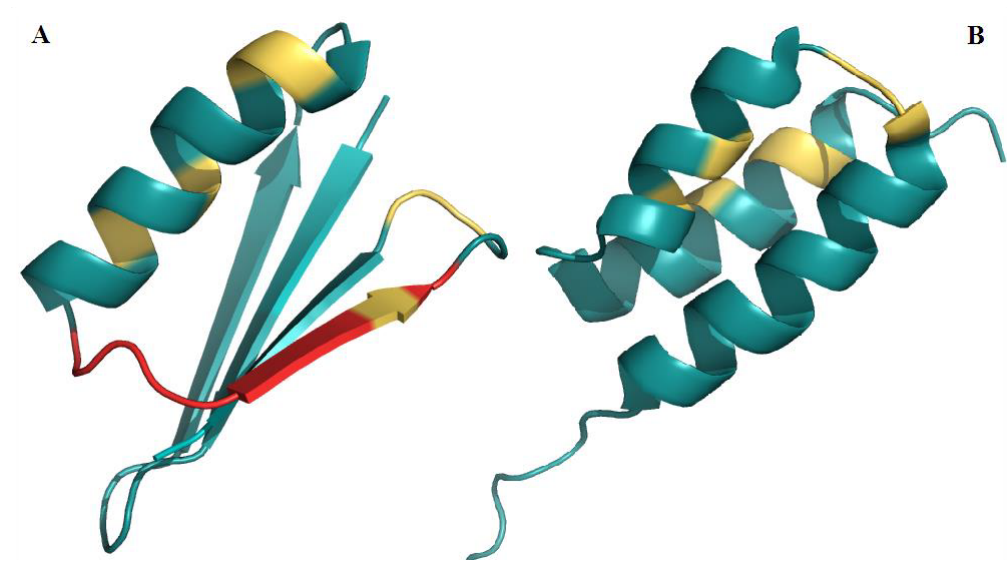

Colors indicate the status of each fragment: red area diverges from the model while blue ones remain consistent with it. Yellow fragments mark the loci of mutations Figure 3: 3D protein structure model. A) 2JWU, B) 2JWS. 


\begin{tabular}{|c|c|c|c|c|}
\hline & SECONDARY FRAGMENT & 이 & 이R & RD \\
\hline A219 (1ZXH) & COMPLETE MOLECULE & 0.085 & 0.189 & 0.310 \\
\hline Mut 1 & B 1-9 & 0.111 & 0.124 & 0.471 \\
\hline Mut 4 & B 12-20 & 0.059 & 0.134 & 0.305 \\
\hline Mut 6 & H 22-39 & 0.079 & 0.173 & 0.313 \\
\hline Mut 3 & B 42-44 & 0.101 & 0.279 & 0.266 \\
\hline Mut 4 & L 45-49 & 0.067 & 0.073 & 0.478 \\
\hline \multirow[t]{2}{*}{ Mut 3} & B $50-53$ & 0.255 & 0.180 & 0.056 \\
\hline & B $54-55$ & 0.039 & 0.160 & 0.197 \\
\hline \multirow[t]{2}{*}{ G311 (1ZXG) } & COMPLETE MOLECULE & 0.095 & 0.288 & 0.248 \\
\hline & L 1-7 & 0.087 & 0.196 & 0.308 \\
\hline Mut 2 & H 8-18 & 0.079 & 0.308 & 0.205 \\
\hline Mut 5 & L 19-24 & 0.039 & 0.177 & 0.183 \\
\hline Mut 2 & H 25-37 & 0.070 & 0.262 & 0.211 \\
\hline \multirow[t]{2}{*}{ Mut 11} & H 40-55 & 0.069 & 0.218 & 0.242 \\
\hline & L 56-59 & 0.100 & 0.493 & 0.168 \\
\hline
\end{tabular}

Table 2: Properties of selected proteins from the point of view of the fuzzy oil drop model. O|T values indicate the distance between the observed and theoretical distribution while O|R values show the corresponding distance between the observed and uniform distribution. RD values are in relation to the idealized (theoretical) distribution. Each parameter is listed for complete molecules and for their individual folds. The leftmost column also includes information on sequential discrepancies between pairs of molecules. Mut- $N$ indicates an exchange and the number of affected residues in each fragment.
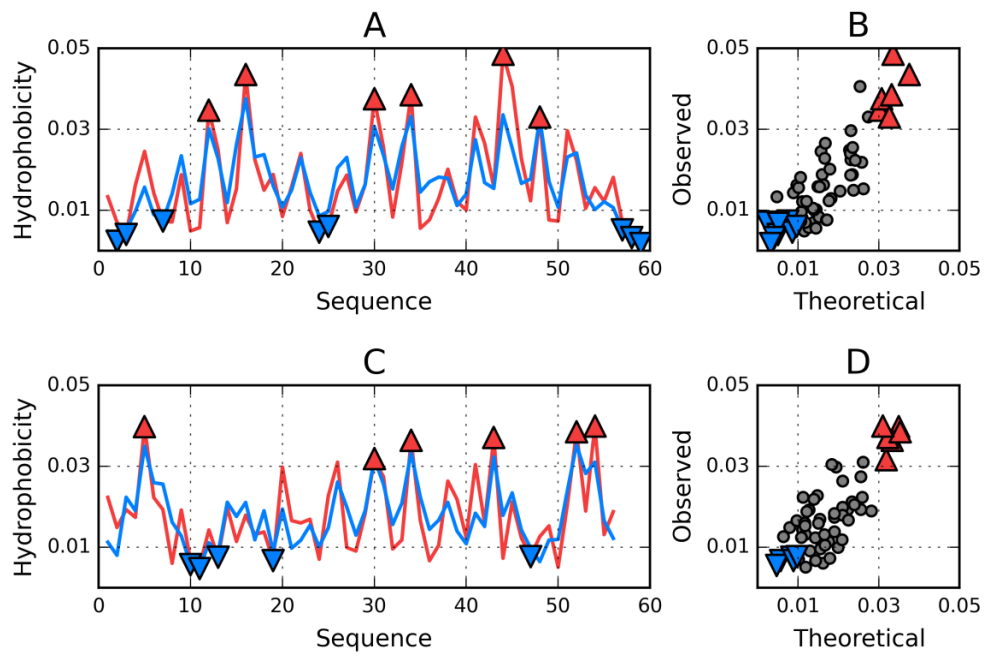

Figure 4: Hydrophobicity density distributions and relations between them: (A and B) G311, (C and D) A219. Red, pointing upwards triangles mark residues accordant with the model and exhibiting high values of both kinds of hydrophobicity. Blue, pointing downwards triangles denote residues also accordant with the models, however presenting low hydrophobicity qualities. Cutoffs of 0.03 and 0.01 were chosen arbitrarily. Both proteins are highly accordant with the model, which also causes high correlation between their hydrophobicity distributions: 0.85 and 0.78 respectively.

hill proteins, which exhibit near-perfect accordance with the theoretical hydrophobicity density distribution [30,31]. The role of these proteins is to be well soluble without any specific interaction with any molecules from environment except water to not allow the ice-structuralization of water.

\section{Homologous proteins}

Two proteins of common ancestry: 2PIJ and 3BD1 are characterized in Table 3. Both of them represent well defined hydrophobic core (distribution of observed hydrophobicity density is similar to expected one-RD below 0.5). Two secondary structural fragments were recognized as locally discordant in 2PIJ and one in 3BD1.

Analysis of $\mathrm{RD}$ values calculated using the OORF system for two homologous proteins reveals differences in the structure of their hydrophobic cores. In 3BD1 nearly the entire chain remains consistent with theoretical predictions (with the exception of several frames in the C-terminal section of the chain). The OORF profile visualize opposite role of certain fragments of the chain. In 2PIJ the central fragment (2030 windows) represent local maximum, while analogical fragment in $3 \mathrm{BD} 1$ reaches its lowest level of $\mathrm{RD}$ values. In $2 \mathrm{PIJ}$ the $\mathrm{RD}$ parameter reaches higher values, especially in the central and the C-terminal fragment of the chain. Both distributions are characterized by low values for the $\mathrm{N}$-terminal fragment (positions 1-10) where the RD parameter does not generally exceed 0.5.

The presence of a complexation partner (marked "P" in Table 3 ) or a ligand ("L") does not seem to affect hydrophobicity density distribution in the relevant areas. In general, whenever ligand interaction requires a large discordant cavity, the corresponding deviation can usually be noted by deficiency of hydrophobicity density which can be identifying 


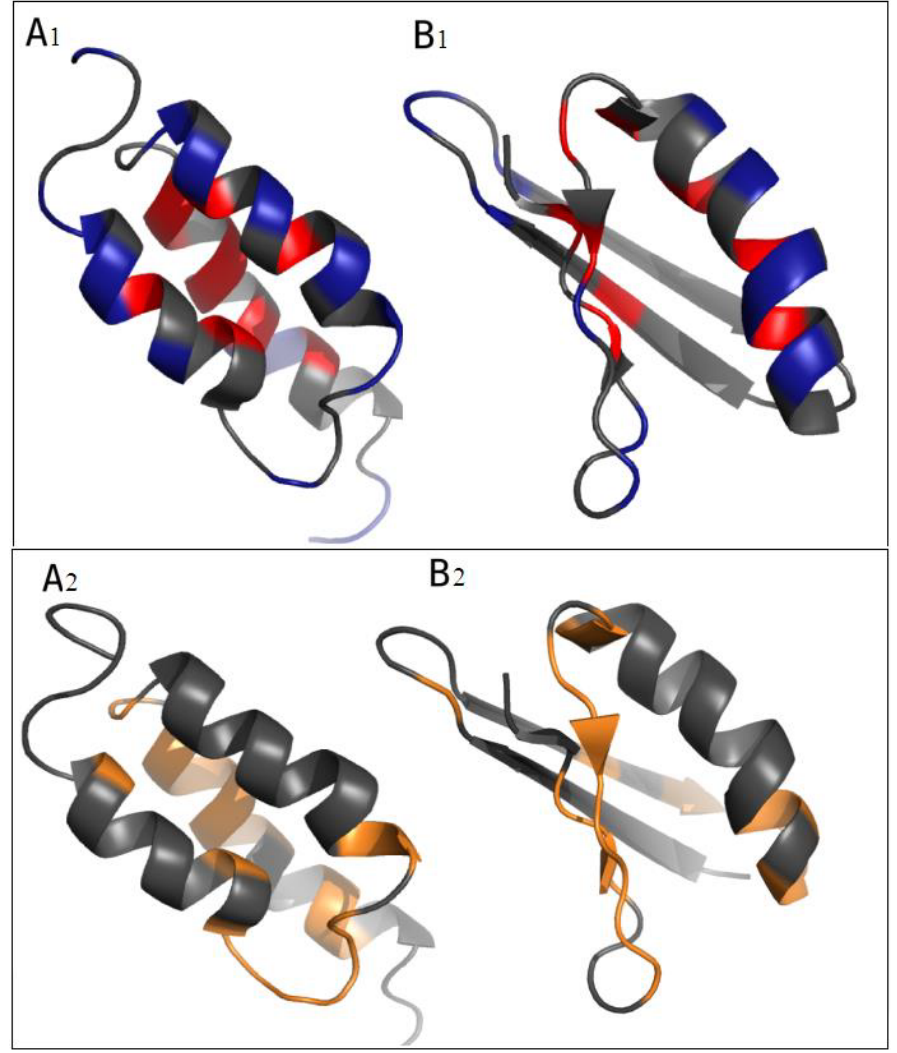

A1-G311-red-hydrophobic core (as marked in Figure 4A). B1-A219-red-hydrophobic core (as marked in Figure 4C) A2-G311-orange-residues differing (mutations) from those in A219. B2-A219-orange-residues differing (mutations) from those in G311.

Figure 5: 3D presentation of $A 219$ and $\mathrm{G} 311$ molecules with highlighted residues.

\begin{tabular}{|c|c|c|c|c|}
\hline & SECONDARY FRAGMENT & 이 & O|lR & RD \\
\hline \multirow[t]{7}{*}{ 2PIJ } & COMPLETE MOLECULE & 0.118 & 0.123 & 0.489 \\
\hline & B 2-5 & 0.088 & 0.161 & 0.356 \\
\hline & $\mathrm{H}-6-13$ & 0.023 & 0.194 & 0.105 \\
\hline & H 15-24 & 0.077 & 0.034 & 0.689 \\
\hline & H 26-36 & 0.036 & 0.044 & 0.453 \\
\hline & B $39-45$ & 0.105 & 0.178 & 0.370 \\
\hline & B 49-55 & 0.077 & 0.046 & 0.625 \\
\hline \multirow[t]{4}{*}{ 3BD1 } & COMPLETE MOLECULE & 0.065 & 0.085 & 0.433 \\
\hline & $\begin{array}{c}\text { A } \\
\mathrm{H} 2-12\end{array}$ & 0.042 & 0.057 & 0.420 \\
\hline & H 13-22 & 0.025 & 0.014 & 0.646 \\
\hline & H 24-35 & 0.030 & 0.098 & 0.236 \\
\hline$L, P$ & H 38-41 & 0.035 & 0.058 & 0.378 \\
\hline$P$ & H $42-49$ & 0.030 & 0.067 & 0.309 \\
\hline \multirow[t]{2}{*}{ L } & H 54-59 & 0.047 & 0.070 & 0.403 \\
\hline & L 50-53 & 0.014 & 0.091 & 0.135 \\
\hline
\end{tabular}

Table 3: Comparison of fuzzy oil drop parameters for homologous proteins. O|T, O|R and RD values indicate the status of each molecule and each of its secondary structural fragments. " $\mathrm{L}$ " and "P" codes appearing in the leftmost column indicate involvement in ligand binding and protein complexation respectively. $\mathrm{H}$ and $\mathrm{B}$ denote helical and $\beta$-structural secondary fragments respectively.

on the distribution profile (which is not the case here) [32]. Similarly, protein complexation often occurs in areas of excess hydrophobicity exposed on the protein surface - which, again, is not the case with the presented protein [33].
By comparing the results presented in Table 3 and Figure 6, we can conclude that $3 \mathrm{BD} 1$ possesses a more stable structure, resembling the idealized "fuzzy oil drop" (i.e., with limited differences between the 
observed and theoretical hydrophobicity density distributions). Figure 7 reveals variations in the status of each fold.

Colors indicate the status of each fragment: red areas diverge from the model while cyan ones remain consistent, as shown in Table 3.

\section{Unrelated proteins of common fold}

Two proteins: $3 \mathrm{CHY}$ wild-type CheY from Escherichia coli, where residue Asp-57 (supported by Lys-109) undergoes phosphorylation and $1 \mathrm{RCF}$ - oxidized recombinant flavodoxin from the cyanobacterium Anabaena 7120 responsible for electron transfer from photosystem I to ferredoxin-NADP $(+)$ reductase [22].

The distributions of expected and observed hydrophobicity density distribution in both proteins reveal the high similarity between these two profiles. It is also expressed by low values of $\mathrm{RD}: 0.300(\mathrm{O} \mid \mathrm{T}=0.089$, $\mathrm{O} \mid \mathrm{R}=0.207)$ for $1 \mathrm{RCF}$ and $\mathrm{RD}=0.443(\mathrm{O}|\mathrm{T}=1.147, \mathrm{O}| \mathrm{R}=0.185)$ for $3 \mathrm{CHY}$. However the secondary fragments representing the status of $\mathrm{RD}>0.5$ were found. The helical fragment (112-128) in 3CHY appeared to represent the status discordant versus the model as well as the loop (76-81). Two $\alpha$-structural fragments $(48-54,120-122)$ in $1 \mathrm{RCF}$ represent the status discordant versus the model as well as the loop 9098 (Figure 8).

The location of fragments representing the distribution of hydrophobicity density in $3 \mathrm{D}$ structure of both proteins appeared to be different. The fragments placed rather on the surface of protein in $3 \mathrm{CHY}$ represent the discordant status while in $1 \mathrm{RCF}$ the dissimilarity versus the model is occurring in the central part of the molecule (Figures 8A8D). This observation may suggest different instability of these two molecules, assuming that other than regular ordered hydrophobicity density distribution may influence the local stability. The biological activity of $3 \mathrm{CHY}$ requires complexation with other protein molecule [21]. The discordance identified on the protein surface suggests potential area ready for complexation as may be concluded from the FOD model.

Local instability (local discordance observation versus $\beta$-expectation) in this case implies substantially different potential tendency to structural differentiation. The lower stability may be supposed in 1RCF as the disagreement is localized in the core of the molecule makes the structural less stable in comparison to analogical $\beta$-structural part in 3CHY (Figure 9). It may suggest the easier destabilization of entire molecule (1RCF) while the stable core in $3 \mathrm{CHY}$ may protect the molecule against decomposition of the central part of the molecule.

One shall note that the divergence entropy used to measure the differences between profiles recognizes as different positions of opposite tendency. Even large surface between profiles may be ignored by divergence entropy calculation as long as two profiles represent similar tendency.

Green space-filling-residues engaged in biological activity (according to Volz and Matsumura [21])

Yellow fragments-RD above 0.5 recognized according to OORF calculation.

Red fragments-discordant fragments according to RD calculated
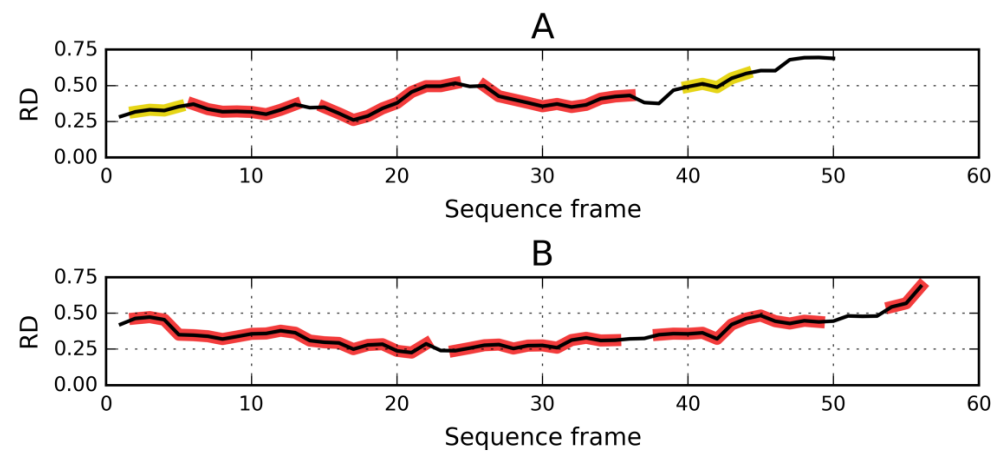

Figure 6: Distribution of RD values calculated using the OORF system (10 aa Overlapped Open Reading Frame) for two homologous proteins: (A) 2PIJ and (B) 3BD1. Red and yellow fragments denote locations within the sequence of $\alpha$-helices and $\beta$-sheets, respectively.

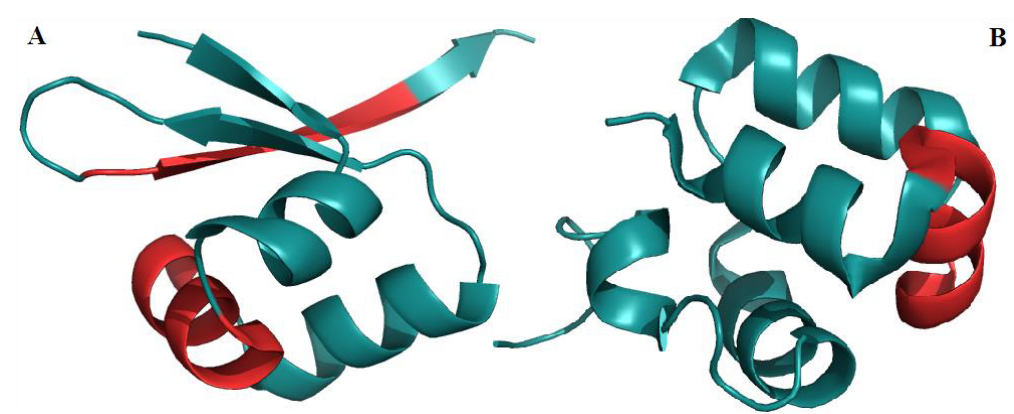

Figure 7: 3D structure of homologous proteins. A) 2PIJ, B) 3BD1. 

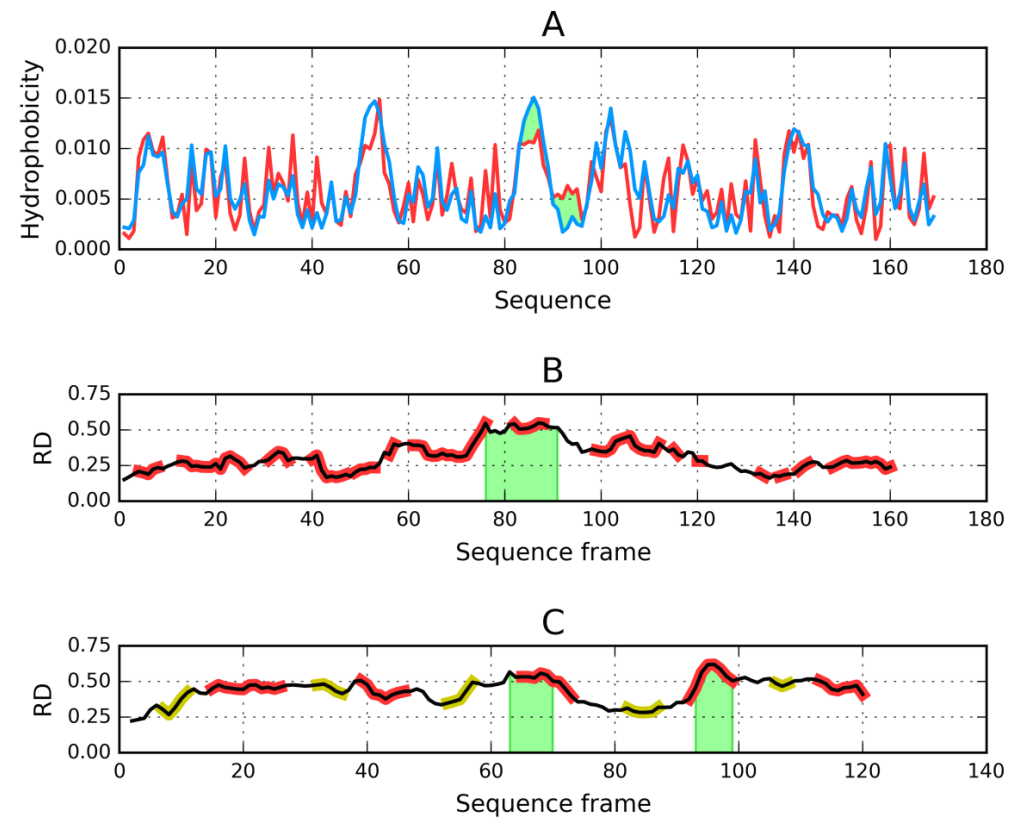

D

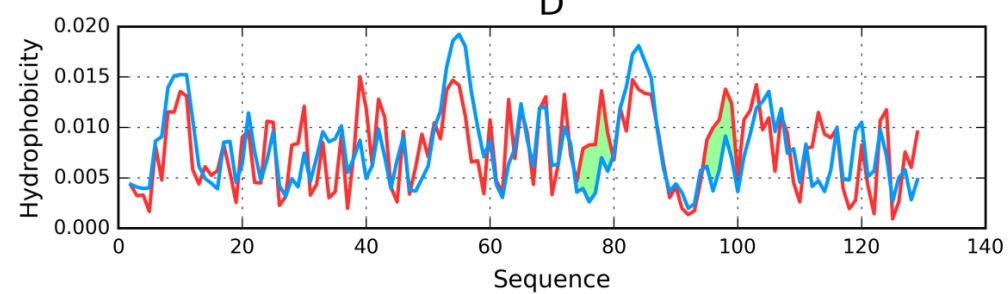

Figure 8: Profiles of theoretical (blue) hydrophobicity, observed (red) hydrophobicity and RD (black) values in 10 aa OORF system distributions: (A and B) $1 \mathrm{RCF}$ $(\mathrm{C}$ and $\mathrm{D}) 3 \mathrm{CHY}$. Green shades between/under the profiles indicate local hydrophobicity discrepancies. Meaning of red and yellow fragments is the same as in Figures 2 and 6.

A

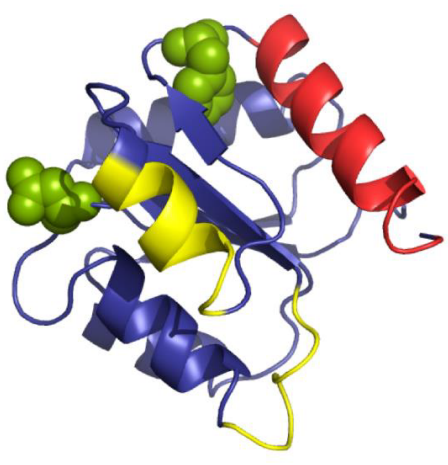

B

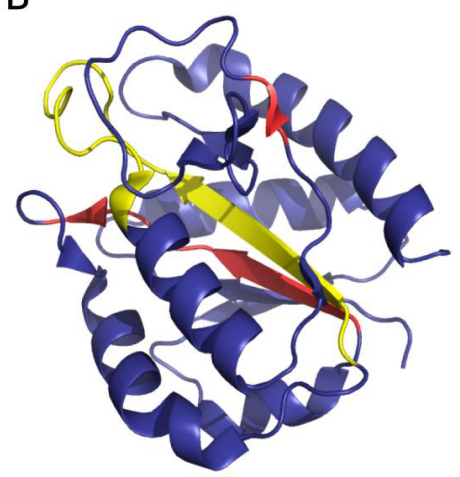

Figure 9: 3D presentation of $(A) C H Y$ and $(B) 1 R C F$.

for secondary fragments.

These two proteins not related one to the other (sequence similarity of only 19\% (Clustal2.1 calculation with standard parameters) shows that the structural similarity does not necessarily ensures similar stability of the protein taken the interpretation of FOD model as the criteria for stability estimation.

\section{Discussion and Conclusion}

The study of sequence-to-structure correlations in proteins has a long history [34]. This work hints at the importance of the hydrophobic core in determining the protein's tertiary conformation. 
Our observations support the suggestions contained in Bakker [35], where the authors conclude that protein structure remains tolerant to residue substitutions as long as the hydropathic profile of the sequence is preserved. Since water is an important factor in this process, much effort has been directed towards analyzing the influence of the proteins' aqueous environment.

They way in which residue sequences encode 3D structures remain a fundamental question in biology. One approach to understanding the folding process is to design a pair of proteins with maximum sequence identity but with differing folds. Therefore, the nonidentity's must be responsible for determining which fold topology prevails and constitute a fold-specific folding code. The intentionally designed proteins $\mathrm{G}_{\mathrm{A}} 88$ and $\mathrm{G}_{\mathrm{B}} 88$, with $88 \%$ sequence identity but different folds and functions [36] are described here in the context of the FOD model. Despite a large number of mutations which together bring sequence identity from $16 \%$ to $88 \%, G_{A} 88$ and $G_{B} 88$ maintain their distinct wild-type 3-alpha and alpha/beta folds, respectively. As the Alexander et al. [36] claim, the 3D-structure determination of two monomeric proteins with such high sequence identity but different fold topology is unprecedented. The geometries of seven nonidentical residues (of 56 total) provide insight into the structural basis for switching between $3-\alpha$ and $\alpha / \beta$ conformations. The FOD model applied to these two de novo designed proteins, to two wild-type proteins with intentionally modified sequences as well as to two homologous proteins, reveals the importance of hydrophobic core structure. Our analysis proves that the hypothesis expressing the dominant role of hydrophobic interactions in tertiary structural stabilization can be confirmed quantitatively. Additionally the role of hydrophobic core in stabilization of structures of natural proteins modified (mutated) to converge to a point of highlevel sequence identity while retaining their respective wild-type tertiary folds, of natural proteins with common ancestry but with differing structures and biological profiles shaped by divergent evolution as well as of natural proteins of high structural similarity with no sequence similarity and different biological function was recognized as main mechanism for structure stabilization as expressed by FOD model.

The FOD model posits a structure which consists of a hydrophobic core (central part of the protein body) together with a sheath acting as a buffer zone between the hydrophobic center and the hydrophilic surface. The role of water in the protein folding process and its influence on the final structure of the protein remains a persistent subject in molecular biology [34,37]; however, the question of generalizing the presented observations remains an open issue. The application of FOD model for amyloidosis mechanism is presented Roterman et al. [29]. The applicability of FOD was tested on few selected sets of proteins of small size [38], structural similarity [39], protein complexes [40] and intrinsically disordered proteins [41].

\section{References}

1. Giri R, Morrone A, Travaglini-Allocatelli C, Jemth P, Brunori M, et al. (2012) Folding pathways of proteins with increasing degree of sequence identities but different structure and function. Proc Natl Acad Sci 109: 17772-17776.

2. Ambroggio XI, Kuhlman B (2009) Computational design of a single amino acid sequence that can switch between two distinct protein folds. J Am Chem Soc 128: 1154-1161.

3. Anderson TA, Cordes MH, Sauer RT (2005) Sequence determinants of a conformational switch in a protein structure. Proc Natl Acad Sci 102: 1834418349

4. Löytynoja A, Goldman N (2008) A model of evolution and structure for multiple sequence alignment. Philos Trans R Soc Lond B Biol Sci 363: 3913-3919.

5. Davidson AR (2008) A folding space odyssey. Proc Natl Acad Sci 105: 2759-
2760.

6. Dalal S, Regan L (2000) Understanding the sequence determinants of conformational switching using protein design. Protein Sci 9: 1651-1659.

7. Copley RR, Russell RB, Ponting CP (2001) Sialidase like Asp-boxes: Sequencesimilar structures within different protein folds. Protein Sci 10: 285-292.

8. Prudhomme N, Chomilier J (2009) Prediction of the protein folding core: application to the immunoglobulin fold. Biochimie 91: 1465-74.

9. Banach M, Prudhomme N, Carpentier M, Duprat E, Papandreou N, et al. (2015) Contribution to the prediction of the fold code: application to immunoglobulin and flavodoxin cases. PLoS ONE 10: e0125098.

10. Banach M, Konieczny L, Roterman I (2014) The fuzzy oil drop model, based on hydrophobicity density distribution, generalizes the influence of water environment on protein structure and function. J Theor Biol 359: 6-17.

11. Hansen N, Allison JR, Hodel FH, van Gunsteren WF (2013) Relative Free Enthalpies for Point Mutations in Two Proteins with Highly Similar Sequences but Different Folds. Biochemistry 52: 4962-4970.

12. Zhou X, Alber F, Folkers G, Gonnet GH, Chelvanayagam G (2000) An analysis of the helix-to-strand transition between peptides with identical sequence. Proteins 41: 248-256.

13. Mattevi A, Valentini G, Rizzi M, Speranza ML, Bolognesi M, et al. (1995) Crysta structure of Escherichia coli pyruvate kinase type I: molecular basis of the allosteric transition. Structure 3: 729-741.

14. Kobe B (1999) Autoinhibition by an internal nuclear localization signal revealed by the crystal structure of mammalian importin alpha. Nat Struct Biol 6: 388-397.

15. Jacoboni I, Martelli PL, Fariselli P, Compiani M, Casadio R (2000) Predictions of protein segments with the same aminoacid sequence and different secondary structure. A benchmark for predictive methods Proteins: Structure, Function, and Bioinformatics 41: 535-544.

16. Krissinel E (2007) On the relationship between sequence and structure similarities in proteomics. Bioinformatics 23: 717-723.

17. He Y, Chen Y, Alexander P, Bryan PN, Orban J (2008) NMR structures of two designed proteins with high sequence identity but different fold and function Proc Natl Acad Sci USA 105: 14412-14417.

18. Sillitoe I, Cuff AL, Dessailly BH, Dawson NL, Furnham N, et al. (2013) New functional families (FunFams) in CATH to improve the mapping of conserved functional sites to 3D structures. CATH - Nucleic Acids Res 41: D490-D498.

19. He Y, Yeh DC, Alexander P, Bryan PN, Orban J (2005) Solution NMR structures of IgG binding domains with artificially evolved high levels of sequence identity but different folds. Biochemistry 44: 14055-14061.

20. Roessler GG, Hall BM, Anderson WJ, Ingram WM, Roberts SA, et al. (2008) Transitive homology-guided structural studies lead to discovery of Cro proteins with $40 \%$ sequence identity but different folds. Proc Natl Acad Sci U S A 105: 2343-2348.

21. Volz K, Matsumura $P$ (1991) Crystal structure of Escherichia coli CheY refined at 1.7-A resolution. J Biol Chem 266: 15511-15519.

22. Burkhart BM, Ramakrishnan B, Yan H, Reedstrom RJ, Markley JL, et al. (1995) Structure of the trigonal form of recombinant oxidized flavodoxin from Anabaena 7120 at 1.40 A resolution. Acta Crystallogr D Biol Crystallogr 51: 318-330.

23. Konieczny L, Bryliński M, Roterman I (2006) Gauss-function-based model of hydrophobicity density In proteins. In Silico Biol 6: 15-22.

24. Kalinowska B, Banach M, Konieczny L, Roterman I (2015) Application of Divergence Entropy to Characterize the Structure of the Hydrophobic Core in DNA Interacting Proteins. Entropy 17: 1477-1507.

25. Kauzmann W (1959) Some factors in the interpretation of protein denaturation Adv Protein Chem 14: 1-63.

26. Levitt MA (1976) A simplified representation of protein conformations for rapie simulation of protein folding. J Mol Biol 104: 59-107.

27. Kullback S, Leibler RA (1951) On information and sufficiency. Ann Math Stat 22: 79-86.

28. de Beer TAP, Berka K, Thornton JM, Laskowski RA (2014) PDBsum additions Nucleic Acids Res 42: D292-D296.

29. Roterman I, Banach M, Kalinowska B, Konieczny L (2016) Influence of 
Citation: Banach M, Kalinowska B, Konieczny L, Roterman I (2016) Structural Role of Hydrophobic Core in Proteins-Selected Examples. J Proteomics Bioinform 9: 276-286. doi: 10.4172/jpb.1000416

aqueous environment on protein structure-plausible hypothesis concerning the mechanism of amyloidogenesis. Entropy 18: 351.

30. Banach M, Prymula K, Jurkowski W, Konieczny L, Roterman I (2012) Fuzzy oil drop model to interpret the structure of antifreeze proteins and their mutants. J Mol Model 18: 229-237.

31. Roterman I, Konieczny L, Jurkowski W, Prymula K, Banach M (2011) Twointermediate model to characterize the structure of fast-folding proteins. J Theor Biol 283: 60-70.

32. Banach M, Konieczny L, Roterman I (2012) Ligand-binding site recognition. In: Irena Roterman-Konieczna (ed.) Protein folding in silico. Woodhead Publishing, New Delhi pp: 79-94.

33. Banach M, Konieczny L, Roterman I (2012) Use of the "fuzzy oil drop" model to identify the complexation area in protein homodimers. In: Roterman-Konieczna I (ed.) Protein folding in silico. Woodhead Publishing, New Delhi, pp: 95-122.

34. Lesk AM, Chothia C (1980) The structure and evolutionary dynamics of the globins. How different amino acid sequences determine similar protein structures. J Mol Biol 136: 225-230.

35. Bakker HJ (2012) Water's response to the fear of water. Nature 491: 533-535.

36. Alexander PA, He Y, Chen Y, Orban J, Bryan PN (2007) The design and characterization of two proteins with $88 \%$ sequence identity but different structure and function. PNA 104: 11963-11968.

37. Davis JG, Gierszal KP, Wang P, Ben-Amotz D (2012) Water structural transformation at molecular hydrophobic interfaces. Nature 491: 582-585.

38. Prymula K, Sałapa K, Roterman I (2010) "Fuzzy oil drop" model applied to individual small proteins built of 70 amino acids. J Mol Model 16: 1269-1282.

39. Banach M, Roterman I, Prudhomme N, Chomilier J (2014) Hydrophobic core in domains of immunoglobulin-like fold. J Biomol Struct Dyn 32: 1583-1600.

40. Piwowar M, Banach M, Konieczny L, Roterman I (2014) Hydrophobic core formation in protein complex of cathepsin. J Biomol Struct Dyn 32: 1023-1032.

41. Kalinowska B, Banach M, Konieczny L, Marchewka D, Roterman I (2014) Intrinsically disordered proteins-relation to general model expressing the active role of the water environment. Adv Protein Chem Struct Biol 94: 315-346. 